09.4

\title{
Поляризуемость наночастиц металлов в телекоммуникационном диапазоне длин волн
}

\author{
() А.И. Сидоров ${ }^{1,2}$, А.И. Сивак ${ }^{1}$, Н.В. Вакула ${ }^{1}$ \\ ${ }^{1}$ Университет ИтМО, Санкт-Петербург, Россия \\ ${ }^{2}$ Санкт-Петербургский государственный электротехнический университет „ЛЭтИ“, Санкт-Петербург, Россия \\ E-mail: sidorov@oi.ifmo.ru
}

Поступило в Редакцию 9 января 2020г.

В окончательной редакции 9 января 2020г.

Принято к публикации 12 марта 2020г.

\begin{abstract}
Проведено численное моделирование поляризуемости и сечения поглощения металлических $(\mathrm{Ag}, \mathrm{Au}, \mathrm{Cu}$ и $\mathrm{Na}$ ) наночастиц в стекле вдали от плазмонного резонанса, в спектральном интервале $1-1.6 \mu \mathrm{m}$. На примере наночастиц Ag показано, что в телекоммуникационном диапазоне длин волн сечение поглощения наночастиц уменьшается в $10^{3}$ раз по сравнению с аналогичной величиной в области плазмонного резонанса. В то же время поляризуемость уменьшается лишь в 10 раз. Проведено сравнение поляризуемостей наночастиц из указанных материалов, имеющих разную геометрию. Показана перспективность применения металлических наночастиц для создания электрооптических стекол.
\end{abstract}

Ключевые слова: наночастица, поляризуемость, сечение поглощения, электрооптическое стекло.

DOI: 10.21883/PJTF.2020.11.49496.18202

Электрооптические эффекты широко используются для управления оптическими сигналами в системах телекоммуникаций и обработки информации. Как правило, в электрооптических устройствах используются электрооптические кристаллы [1], такие как $\mathrm{LiNbO}_{3}$, $\mathrm{BaTiO}_{3}$ и др. Однако технологии их выращивания и обработки сложны. В настоящее время созданы дешевые электрооптические стеклокерамики, содержащие электрооптические нано- и микрокристаллы [2,3]. Из таких стеклокерамик могут быть изготовлены различные оптические элементы, в том числе оптические волокна. Основным их недостатком является повышенное светорассеяние. Атомы и ионы тяжелых металлов обладают относительно высокой электронной поляризуемостью. Поэтому стекла, содержащие такие атомы или ионы, также предлагаются в качестве электрооптических материалов [4,5]. Металлические наночастицы (НЧ) обладают значительно большей поляризуемостью, чем ионы тяжелых металлов, особенно в спектральном интервале, соответствующем плазмонному резонансу [6,7], который лежит в видимой или ультрафиолетовой областях спектра. Однако в области плазмонного резонанса сечение поглощения металлических НЧ резко возрастает. Вдали от плазмонного резонанса, например в телекоммуникационном диапазоне длин волн, сечение поглощения уменьшается, а поляризуемость НЧ остается относительно высокой. В неорганических стеклах и кристаллах могут быть синтезированы металлические НЧ из разных металлов ( $\mathrm{Ag}, \mathrm{Au}, \mathrm{Cu}, \mathrm{Na}$ и др.), имеющие различную форму, размеры и структуру [8-11]. В связи с этим изучение поляризуемости металлических НЧ в стеклах вдали от плазмонного резонанса представляет как научный, так и практический интерес. Электрооптический коэффициент композитных материалов зависит от концентрации активных центров и их поляризуемости. Поэтому в настоящей работе поставлена задача изучения методами численного моделирования влияния материала и геометрии металлических НЧ на их поляризуемость в стекле в спектральном интервале $1-1.6 \mu \mathrm{m}$.

Численное моделирование проводилось для НЧ, размер которых много меньше длины волны излучения. В этом случае для расчета поляризуемости НЧ может быть использовано дипольное квазистатическое приближение $[6,7,12]$. В данном приближении поляризуемость $\delta$ описывается следующими выражениями: для сферической НЧ

$$
\delta=2 \pi r^{3} \frac{\varepsilon_{p}-\varepsilon_{h}}{\varepsilon_{p}+2 \varepsilon_{h}},
$$

где $r$ - радиус НЧ, $\varepsilon_{p}-$ диэлектрическая проницаемость НЧ, $\varepsilon_{h}$ - диэлектрическая проницаемость окружающей среды;

для сферической НЧ с диэлектрической оболочкой

$$
\begin{gathered}
\delta=2 \pi r_{s}^{3} \frac{\varepsilon_{s} \varepsilon_{a}-\varepsilon_{h} \varepsilon_{b}}{\varepsilon_{s} \varepsilon_{a}+2 \varepsilon_{h} \varepsilon_{b}}, \\
\varepsilon_{a}=\varepsilon_{c}(3-2 P)+2 \varepsilon_{s} P, \\
\varepsilon_{b}=\varepsilon_{c}+\varepsilon_{s}(3-P), \quad P=1-\left(\frac{r_{c}}{r_{s}}\right)^{3},
\end{gathered}
$$

где $\varepsilon_{h}, \varepsilon_{c}, \varepsilon_{s}$ - диэлектрические проницаемости среды, ядра и оболочки наночастицы соответственно, $r_{c}-$ радиус ядра, $r_{s}$ - радиус оболочки;

для наносфероида (эллипсоида вращения)

$$
\delta_{i}=4 a^{2} b \frac{\varepsilon_{p}-\varepsilon_{h}}{3 \varepsilon_{h}+3 L_{i}\left(\varepsilon_{p}-\varepsilon_{h}\right)} .
$$




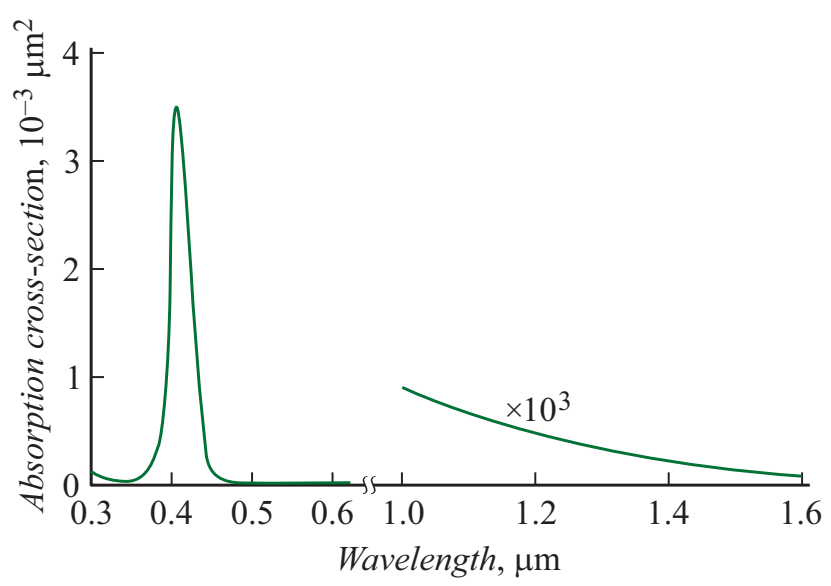

Рис. 1. Спектральная зависимость сечения поглощения сферической наночастицы из серебра.

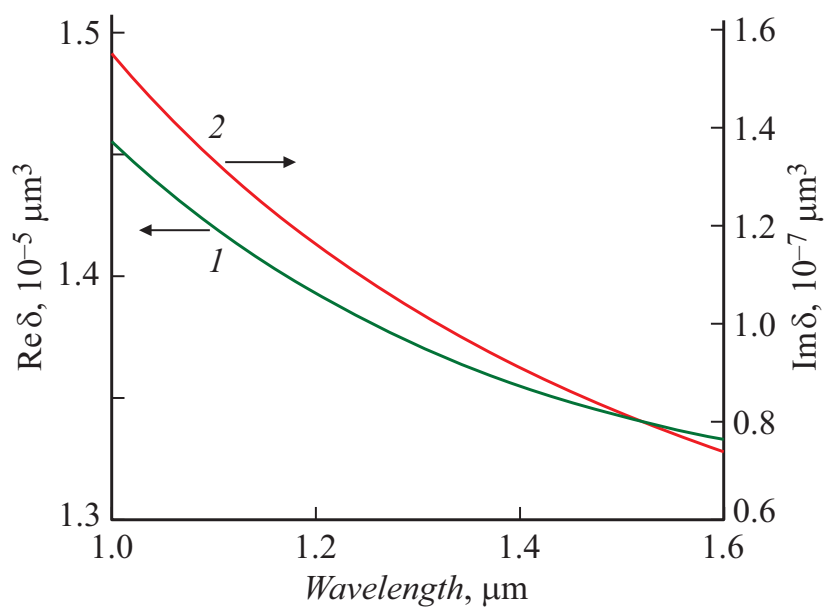

Рис. 2. Спектральная зависимость действительной (1) и мнимой (2) частей поляризуемости сферической наночастицы из серебра в стекле.

Здесь индекс $i=1,2$ соответствует ориентации электромагнитного поля вдоль осей $a$ и $b$ сфероида, $\varepsilon_{p}$ и $\varepsilon_{h}$ - диэлектрические проницаемости материалов сфероида и среды соответственно, $L$ - геометрический фактор,

$$
L_{i}=\frac{a^{2} b}{2} \int_{0}^{\infty} \frac{d x}{\left(z_{i}^{2}+x\right) \sqrt{\left(a^{2}+x\right)\left(b^{2}+x\right)\left(c^{2}+x\right)}}, z_{i}=\left(\begin{array}{l}
a \\
b
\end{array}\right) .
$$

Сечение поглощения НЧ $\sigma_{a}$ описывается следующим выражением:

$$
\sigma_{a}=k \operatorname{Im} \delta,
$$

где $k$-волновое число $(k=2 \pi / \lambda), \lambda$ - длина волны излучения. Из приведенных выражений видно, что поляризуемость НЧ пропорциональна объему НЧ и зависит как от диэлектрической проницаемости НЧ, так и от диэлектрической проницаемости окружающей среды. Влияние длины волны проявляется через дис- персию оптических констант. Моделирование проводилось для $\mathrm{HЧ} \mathrm{Ag}, \mathrm{Au}, \mathrm{Cu}$ и $\mathrm{Na}$ радиусом $r=10 \mathrm{~nm}$ с учетом дисперсии их оптических констант [13-15]. Показатель преломления силикатного стекла $n=1.52$. Показатель преломления оболочки для НЧ с оболочкой $n=2.2(\mathrm{AgCl})$. Расчеты проводились для наносфероидов с $a=10 \mathrm{~nm}$ и $b=20 \mathrm{~nm}$.

На рис. 1 показана спектральная зависимость сечения поглощения сферической НЧ из серебра в стекле. В спектре присутствует узкая полоса поглощения на длине волны $\lambda=0.41 \mu \mathrm{m}$, соответствующая плазмонному резонансу НЧ серебра. В спектральном интервале $1-1.6 \mu \mathrm{m}$ сечение поглощения НЧ более чем на три порядка меньше величины в области плазмонного резонанса. Плазмонные резонансы сферических НЧ из Аu, $\mathrm{Cu}$ и $\mathrm{Na}$ в стекле находятся на длинах волн 0.53, 0.56 и $0.51 \mu \mathrm{m}$ соответственно.

В качестве примера на рис. 2 показаны спектральные зависимости действительной и мнимой частей поляризуемости сферической НЧ из серебра в стекле для спектрального интервала $1-1.6 \mu \mathrm{m}$. Из рисунка видно, что при увеличении длины волны происходит незначительное уменьшение действительной части поляризуемости. При этом мнимая часть поляризуемости уменьшается примерно в 2 раза. Спектральные зависимости действительной и мнимой частей поляризуемости для НЧ из $\mathrm{Au}, \mathrm{Cu}$ и $\mathrm{Na}$, а также для НЧ других геометрий имеют аналогичный вид.

Таблица 1. Влияние материала сферической НЧ на сечение поглощения $\left(\sigma_{a}\right)$, действительную $(\operatorname{Re} \delta)$ и мнимую $(\operatorname{Im} \delta)$ части поляризуемости НЧ

\begin{tabular}{c|c|c|r|r}
\hline Материал & $\lambda, \mu \mathrm{m}$ & $\sigma_{a}, \mu \mathrm{m}^{2}$ & \multicolumn{1}{c}{$\operatorname{Re} \delta, \mu \mathrm{m}^{3}$} & \multicolumn{1}{c}{$\operatorname{Im} \delta, \mu \mathrm{m}^{3}$} \\
\hline $\mathrm{Ag}$ & 0.41 & $3.6 \cdot 10^{-3}$ & $1.5 \cdot 10^{-4}$ & $2.2 \cdot 10^{-4}$ \\
& 1.0 & $9.2 \cdot 10^{-7}$ & $1.45 \cdot 10^{-5}$ & $1.5 \cdot 10^{-7}$ \\
& 1.6 & $2.8 \cdot 10^{-7}$ & $1.33 \cdot 10^{-5}$ & $7 \cdot 10^{-8}$ \\
$\mathrm{Au}$ & 1.0 & $1 \cdot 10^{-6}$ & $1.46 \cdot 10^{-5}$ & $1.52 \cdot 10^{-7}$ \\
& 1.6 & $2.2 \cdot 10^{-7}$ & $1.34 \cdot 10^{-5}$ & $6 \cdot 10^{-8}$ \\
$\mathrm{Cu}$ & 1.0 & $1.1 \cdot 10^{-6}$ & $1.5 \cdot 10^{-5}$ & $1.8 \cdot 10^{-7}$ \\
& 1.6 & $4.2 \cdot 10^{-7}$ & $1.35 \cdot 10^{-5}$ & $1.1 \cdot 10^{-7}$ \\
$\mathrm{Na}$ & 1.0 & $2.2 \cdot 10^{-6}$ & $1.88 \cdot 10^{-5}$ & $3.5 \cdot 10^{-7}$ \\
& 1.6 & $5 \cdot 10^{-7}$ & $1.46 \cdot 10^{-5}$ & $1.5 \cdot 10^{-7}$
\end{tabular}

Таблица 2. Влияние геометрии НЧ из $\mathrm{Ag}$ на длине волны $1.5 \mu \mathrm{m}$ на сечение поглощения $\left(\sigma_{a}\right)$, действительную $(\operatorname{Re} \delta)$ и мнимую $(\operatorname{Im} \delta)$ части поляризуемости НЧ

\begin{tabular}{c|c|c|c}
\hline Геометрия НЧ & $\sigma_{a}, \mu \mathrm{m}^{2}$ & $\operatorname{Re} \delta, \mu \mathrm{m}^{3}$ & $\operatorname{Im} \delta, \mu \mathrm{m}^{3}$ \\
\hline Сфера & $3 \cdot 10^{-7}$ & $1.35 \cdot 10^{-5}$ & $8 \cdot 10^{-8}$ \\
Сфера с оболочкой & $3.5 \cdot 10^{-7}$ & $2 \cdot 10^{-5}$ & $1 \cdot 10^{-7}$ \\
Наносфероид & & & \\
Длинная ось & $3 \cdot 10^{-6}$ & $5.5 . \cdot 10^{-5}$ & $7 \cdot 10^{-7}$ \\
Короткая ось & $4 \cdot 10^{-7}$ & $2.1 \cdot 10^{-5}$ & $1 \cdot 10^{-7}$
\end{tabular}


Табл. 1 иллюстрирует влияние материала НЧ на ее сечение поглощения и поляризуемость для случая сферических НЧ. Для сравнения в таблице приведены данные характеристики для НЧ из серебра на длине волны плазмонного резонанса $(\lambda=0.41 \mu \mathrm{m})$. Видно, что при переходе из области плазмонного резонанса в телекоммуникационный диапазон сечение поглощения НЧ из серебра уменьшается более чем на три порядка. В то же время действительная часть поляризуемости уменьшается лишь в 10 раз. При этом мнимая часть поляризуемости претерпевает значительное уменьшение в $(1.5-3) \cdot 10^{3}$ раз. Минимальное сечение поглощения в телекоммуникационном диапазоне имеют $\mathrm{HЧ}$ из $\mathrm{Ag}$, максимальное - $\mathrm{HЧ} \mathrm{из} \mathrm{Na}$. НЧ из $\mathrm{Au}$ и $\mathrm{Cu}$ имеют величины поляризуемости, близкие к поляризуемости НЧ из Ag. Максимальную поляризуемость имеют НЧ из $\mathrm{Na}$.

Рассмотрим влияние геометрии НЧ на поляризуемость и сечение поглощения на примере НЧ из $\mathrm{Ag}$ на длине волны $1.5 \mu \mathrm{m}$ (табл. 2). Из таблицы видно, что изменение геометрии НЧ при равных размерах слабо влияет на сечение поглощения НЧ. В то же время при добавлении к сферической НЧ диэлектрической оболочки с высоким показателем преломления или при переходе от сферической НЧ к наносфероиду происходит увеличение поляризуемости. Так, $\operatorname{Re} \delta$ наносфероида вдоль длинной оси в 4 раза больше, чем у сферической НЧ, a $\operatorname{Im} \delta$ наносфероида в сравнении с величиной для сферической НЧ больше в 8.7 раза.

Таким образом, с точки зрения поляризуемости оптимальным материалом НЧ для создания электрооптических стекол в телекоммуникационном диапазоне длин волн является $\mathrm{Na}$, а оптимальной геометрией НЧ является наносфероид. Полученные результаты могут быть использованы при разработке композитных материалов на основе стекол с металлическими НЧ для устройств управления оптическими сигналами.

\section{Финансирование работы}

Работа выполнена при финансовой поддержке Министерства образования и науки РФ (проект 16.1651.2017/4.6).

\section{Конфликт интересов}

Авторы заявляют, что у них нет конфликта интересов.

\section{Список литературы}

[1] Tarafder A., Karmakar B. // Ferroelectrics - material aspects / Ed. M. Lallart. Croatia: InTech, 2011. P. 240-278.

[2] Jain H. // Ferroelectrics. 2004. V. 306. P. 111-119.

[3] Tarafder A., Annapurna K., Chaliha R.S., Tiwari V.S., Gupta P.K., Karmakar B. // J. Alloys Compd. 2010. V. 489. P. 281-287.
[4] Tagantsev D.K., Kazansky P.G., Lipovskii A.A., Maluev K.D. // J. Non-Cryst. Solids. 2008. V. 354. P. 1369 1375.

[5] Jilkova K., Mika M., Kostka P., Lahodny F., Nekvindova P., Jankovsky O., Bures R., Kavanova M. // J. Non-Cryst. Solids. 2019. V. 518. P. 51-56.

[6] Kreibig U., Vollmer M. Optical properties of metal clusters. N.Y.: Springer, 1995. $547 \mathrm{p}$.

[7] Климов В.В. Наноплазмоника. М.: Физматлит, 2009. 480 с. [Klimov V.V. Nanoplasmonics. Singapore: Pan Stanford, 2014. $430 \mathrm{p}$.$] .$

[8] Dubrovin V.D., Ignatiev A.I., Nikonorov N.V., Sidorov A.I., Shakhverdov T.A., Agafonova D.S. // Opt. Mater. 2014. V. 36. P. 753-759.

[9] Demichev I.A., Nikonorov N.V., Sidorov A.I. // J. Phys. Chem. C. 2015. V. 119. P. 19344-19349.

[10] Bochkareva E.S., Nikonorov N.V., Podsvirov O.A., Prosnikov M.A., Sidorov A.I. // Plasmonics. 2016. V. 11. P. 241-246.

[11] Bochkareva E.S., Sidorov A.I., Yurina U.V., Podsvirov O.A. // Nucl. Instrum. Meth. Phys. Res. B. 2017. V. 403. P. 1-6.

[12] Bohren C.F., Huffman D.R. Absorption and scattering of light by small particles. N.Y.: John Wiley \& Sons, 1983. 544 p.

[13] Inagaki T., Arakawa E.T., Birkhoff R.D., Williams M.W. // Phys. Rev. B. 1976. V. 13. P. 5610-5612.

[14] Schulz L.G. // J. Opt. Soc. Am. 1954. V. 44. P. 357-362.

[15] Palik E.D. Handbook of optical constants of solids. San Diego: Academic press, 1998. V. 3. 470 p. 